\title{
CPSO algorithm based parameter optimization for power system damping controllers
}

\author{
Wang $\mathrm{Xi}^{1, \mathrm{a}}$, Hu Pengfei ${ }^{1, \mathrm{~b}}$ \\ ${ }^{1}$ Sichuan Electrical Engineering Research Institute of State Grid, Chengdu 610000, China \\ aemail:wangxi_55@sina.cn, bemail:hupengfei01@163.com
}

Keywords: power system; damping controller; CPSO (chaotic particle swarm optimization);

\begin{abstract}
Wide-area PSS (power system stabilizer) and HVDC (High voltage direct current) modulation are effective damping controllers in power system. With conventional parameter tuning method, the damping of the non-dominate modes would be impaired or new poor damping mode would be triggered. In this paper, the CPSO (chaotic particle swarm optimization) algorithm is used to tune the parameters of damping controller. The aim of the optimization is to provide desired damping ratio for all oscillation modes. A case study verifies the effectiveness of the proposed parameter optimization method.
\end{abstract}

\section{Introduction}

With the increasing interconnection of power system, damping of inter-area oscillation plays a more and more important role in increasing the transmission capability and stabilizing the power system. Local damping controllers such as conventional PSS cannot provide enough damping since the lack of global observability. The development of wide-area measurement systems (WAMS) provides a more effective control strategy for inter-area oscillations damping. PSS with suitable wide-area input signals is proved to be effective mean to improve system dynamic performance in terms of better damping of inter-area oscillations [1-3]. HVDC (High Voltage Direct Current) has good controllability and fast response. It has been widely used in long distance power transmission. The HVDC modulation is a unique damping resource in an AC/DC hybrid transmission system. It has inherent advantages in damping inter-area mode since HVDC always serves as tie line of different areas [4,5]. However, in an AC/DC weakly interconnected system, destabilizing interaction between uncoordinated damping controllers can cause dynamic instability [6]. Therefore, a high quality of coordination control strategy is required in AC/DC interconnected system. The basic approaches for designing coordinated damping control system are tuning controller parameters. Pole placement is the most widely-used parameter tuning approach, while the aim of pole placement is to improve the damping ratio of the dominate mode, numerous of parameter combinations could reach the aim. If inappropriately parameter combination is adopted, the damping of the non-dominate modes would be impaired or new poor damping mode would be triggered.

In this paper, we will propose a practical optimization algorithm to design damping controller in an AC/DC interconnected system, the aim of strategy is to enhancing the damping performance of the system. In the proposed strategy, the damping controller parameters are optimized by Chaotic Particle Swarm Optimization (CPSO) Algorithm. With optimized parameters, the overall damping performance of the interconnected system can be further enhanced. The proposed strategy can effectively damping the inter-area oscillation without impairing the damping of other modes, and it is easy to realize in real system.

This paper is organized as follows. Following this section, The CPSO parameter optimization is presented in Section 2. A detailed case study is performed in Section 3. Finally, the general conclusions are summarized in Section 4. 


\section{CPSO algorithm}

To find out the optimal parameters and maximally improve the overall system performance, CPSO algorithm is implemented for parameter optimization of damping controller. The optimization is intended to improve the damping ratio of all modes to certain values.

The PSO algorithm is a population-based stochastic optimization method and it starts by initializing a population of random solutions called particles and searches for optima by updating generations through the following velocity and position update equations [7].

The velocity update equation

$$
v_{\text {id }}(k+1)=\omega v_{i d}(k)+c_{1} r_{1}\left[\text { pbest }_{i d}(k)-x_{i d}(k)\right]+c_{2} r_{2}\left[\text { gbest }_{d}(k)-x_{\text {id }}(k)\right]
$$

The position update equation

$$
x_{i d}(k+1)=x_{i d}(k)+v_{i d}(k+1)
$$

Where $v_{i d}(k)$ and $x_{i d}(k)$ are the velocity and position of the $i$ th particle for the $d$ th dimension at the $k$ th iteration, respectively. $1 \leq i \leq M, 1 \leq d \leq D, 1 \leq k \leq N$. $\omega$ is an inertia weight, $r_{1}$ and $r_{2}$ are random numbers selected between 0 and $1 . c_{1}$ and $c_{2}$ are positive coefficients between 0 and 2 that is $c_{1}+c_{2} \leqslant 4$. pbest $t_{i d}(k)$ and $g b e s t(k)$ are the best position of the ith particle and the entire swarm for the $d$ th dimension, respectively. They are obtained by using the fitness function and the update mechanism.

Studies by Angeline [8] showed that PSO algorithm may experience inappropriate convergence and fall into the local optima as the number of generations was increased. Chaotic sequences have the characteristics of the chaotic map such as randomness, ergodicity and regularity. The application of chaotic sequences instead of random sequences in PSO is a powerful strategy to diversify the population of particles and to improve the PSO's performance in preventing premature convergence to local minimum [9-11].The logistic map is one of the simplest and easiest chaotic maps to employ in the chaotic PSO (CPSO) algorithm. A logistic map is given as follows

$$
Z_{t}=\alpha Z_{t-1}\left(1-Z_{t-1}\right) \quad t=1,2, \cdots
$$

where $Z_{t}$ is the th chaotic number, $Z_{0}$ is a random number in the interval of $(0,1)$ and $Z_{0} \notin\{0.0,0.25,0.5,0.75,1.0\} . \alpha$ is the control parameter, usually set to 4 in the experiments.

CPSO algorithm are initialized using the logistic map for the initial positions and velocities of the particles,

$$
\begin{cases}x_{i d}(1)=\alpha x_{(i-1) d}(1)\left(1-x_{(i-1) d}(1)\right) & 1 \leq i \leq M \\ v_{i d}(1)=\alpha v_{(i-1) d}(1)\left(1-v_{(i-1) d}(1)\right) & 1 \leq d \leq D\end{cases}
$$

$v_{0 d}(1), x_{0 d}(1)$ are initial positions and velocities to produce the first particle positions and velocities at the first iteration. $v_{0 j}(1), x_{0 j}(1) \notin\{0.0,0.25,0.5,0.75,1.0\}$, and they are random numbers in the interval of $(0,1)$.

Furthermore, a chaotic sequence is generated using the logistic map for the inertia weight, $\omega$.This creates the best balance for the inertia weight during the evolution process between the local and global search processes which results in the best convergence capability and search performance. In a same way, the logistic map is also used in two independent random sequences $r_{1}$ and $r_{2}$ to improve the diversity in the CPSO algorithm solution space.

$$
\begin{cases}\omega_{k}=\alpha \omega_{k-1}\left(1-\omega_{k-1}\right) & \\ r_{1, k}=\alpha r_{1, k-1}\left(1-r_{1, k-1}\right) & 1 \leq k \leq n \\ r_{2, k}=\alpha r_{2, k-1}\left(1-r_{2, k-1}\right) & \end{cases}
$$

Where $\omega_{k}$ is the $k$ th chaotic inertia weight, $r_{1 k}$ and $r_{2 k}$ are the two $k$ th independent chaotic random sequences, $\omega_{0}, r_{1,0}, r_{2,0} \notin\{0.0,0.25,0.5,0.75,1.0\}$, and they are random numbers in the interval of ( 0 , 1).Then, the velocity update equation of the CPSO algorithm is rewritten as follows 


$$
v_{i d}(k+1)=\omega_{k} v_{i d}(k)+c_{1} r_{1, k}\left[\text { pbest }_{i d}(k)-x_{i d}(k)\right]+c_{2} r_{2, k}\left[\operatorname{gbest}(k)-x_{i d}(k)\right]
$$

The CPSO algorithm combines the fast search ability of PSO with the stochastic and ergodicity property of chaos generated by logistic map, which results in a better convergence value.

\section{Validation}

In this section, a detailed case is used to show the simplicity and effectiveness of proposed coordination strategy. The EPRI-36 system is a standard test system from Chinese Electric Power Research Institute (EPRI), which comprises 8 generators and 36 buses. There are 3 areas and in total 25 lines in the test system, Generator- 1 to Generator- 8 are connected with BUS1 to BUS8, respectively. HVDC line is installed between Bus33 and Bus34, running in monopole operation.

The HVDC damping controllers could be represented as the following transfer function.

$$
H(s)=K \frac{s T_{w}}{1+s T_{w}} \frac{1+s T_{1}}{1+s T_{2}} \frac{1+s T_{3}}{1+s T_{4}}
$$

Where $K$ is the constant gain, $T_{w}$ is the washout time constant, $T_{1}, T_{3}$ are the lead time constant and $T_{2}, T_{4}$ are the lag time constant. We use the proposed CPSO algorithm to optimize the control parameters which can insure that each damping ratio of each mode. When tuning parameters, $T_{w}, T_{3}$, $T_{4}$ are usually given certain values. $K, T_{1}, T_{2}$ are the variables.

In order to further validate the effectiveness and robustness of the proposed damping controller and parameter optimization algorithm, a three-phase line-to-ground fault in line 27(between BUS16 and BUS19) is investigated by time-domain simulation. Fig. 1 show the dynamic responses of the test system. It is clear that the oscillation is excited under the case, while the system with proposed damping controller can effectively damping the oscillation, which validates the effectiveness of the proposed strategy.

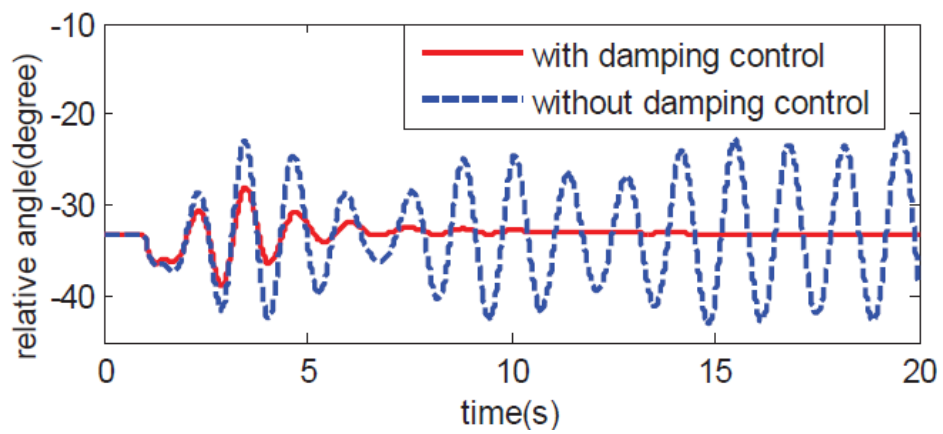

Fig.1 Simulation result

\section{Conclusion}

In this paper, the CPSO (chaotic particle swarm optimization) algorithm is used to tune the parameters of damping controller. The aim of the optimization is to provide desired damping ratio for all oscillation modes. A case study verifies the effectiveness of the proposed parameter optimization method.

\section{Reference}

[1] IEEE Power Engineering Society System Oscillations Work Group, Interarea oscillations in Power system, IEEE 95-TP-101, Oct, 1994.

[2] M. E. Aboul-Ela, A. A. Sallam, J. D. McCalley, A. A. Fouad, Damping controller design for power system oscillations using global signals, IEEE Trans. Power Syst. 11(2) (1996) 767-773.

[3] Zhang Yang, Anjan Bose, Design of wide-area damping controllers for interarea oscillations, IEEE Trans. Power Syst. 23(3) (2008) 1136-1143.

[4] T. Smed, G. Andersson, Utilising HVDC to damp power oscilla-tions, IEEE Trans. Power Del. 
8(1993) 620-627.

[5] Wang Juanjuan, Fu Chuang, Zhang Yao, Design of WAMS-Based Multiple HVDC Damping Control System, IEEE Trans. Smart Grid. 2 (2) (2011) 363-374.

[6] CIGRÉ Task Force 38.02.16, Impact of the interaction among power system controls, Paris, Technical Report. Fall 1999.

[7] Kennedy J, Eberhart RC, Particle swarm optimization, Proc of IEEE Int Conf Neural Networks, iscataway, NJ. (1995) 1942-1948.

[8] P. J. Angeline, Evolutionary optimization versus particle swarm optimization: Philosophy and performance differences, in Proc. 7th Annu. Conf. Evol. Progr. VII. (1998) 601-610.

[9] ALATAS B., AKIN E., OZER A.B, Chaos embedded particle swarm optimization algorithms, J. Chaos, Solitons Fractals. 40 (4) (2009) 1715-1734.

[10] LIU B., WANG L., JIN Y.H., TANG F., HUANG D.X. Improved particle swarm optimization combined with chaos, J. Chaos, Solitons Fractals. 25 (5) (2005) 1261-1271.

[11] D.C. Huynh, M.W. Dunnigan, Parameter estimation of an induction machine using advanced particle swarm optimisation algorithms. IET Electr. Power Appl. 4(9) (2010) 748-760. 